Article

\title{
Development and Evaluation of Multifunctional Poly(Lactic-co-glycolic acid) Nanoparticles Embedded in Carboxymethyl $\beta$-Glucan Porous Microcapsules as a Novel Drug Delivery System for Gefitinib
}

\author{
Xiaonan Li ${ }^{1,2}$, Jinglei Wang ${ }^{2}$, Shang $\mathrm{Li}^{1}{ }^{1}$, Zhaorong Liu ${ }^{2}$, Zhiru Zheng ${ }^{2}$ and Yanzhuo Zhang ${ }^{1,2, *}$ \\ 1 Jiangsu Key Laboratory of New Drug Research and Clinical Pharmacy, Xuzhou Medical University, \\ Xuzhou 221004, China; xiaonanlike@126.com (X.L.); lishang95@126.com (S.L.) \\ 2 Department of Pharmaceutics, School of Pharmacy, Xuzhou Medical University, Xuzhou 221004, China; \\ jinglei94@126.com (J.W.); liuzhaorong123@126.com (Z.L.); xuyynt@126.com (Z.Z.) \\ * Correspondence: yanzhuozhang@126.com; Tel./Fax: +86-516-83262139
}

Received: 23 August 2019; Accepted: 9 September 2019; Published: 12 September 2019

\begin{abstract}
In this study, a new kind of folic acid (FA)-conjugated and chitosan (CS)-coated poloxamer 407 (P407)/poly(lactic-co-glycolic acid) (PLGA) nanoparticles (NPs), FCPP NPs, were prepared, and further micro-encapsulated by carboxymethyl $\beta$-glucan microcapsules (MCs) to produce a multifunctional system of NPs embedded in MCs (NEMs) for potential lung tumor-targeted delivery of gefitinib. The prepared gefitinib-loaded FCPP (GFB/FCPP) NPs showed a hydrodynamic diameter of $255.4 \pm 14.5 \mathrm{~nm}$ and existed in an amorphous state. After encapsulation in carboxymethyl $\beta$-glucan MCs, the GFB/FCPP-based NEMs (GFB/FCPP-NEMs) also exhibited a spherical morphology with a median diameter $\left(\mathrm{d}_{50}\right)$ of around $2.2 \mu \mathrm{m}$. After hydration, GFB/FCPP- NEMs can quickly dissociate into its primary particles, GFB/FCPP NPs. Our in vitro drug release study revealed that these GFB/FCPP-NEMs exhibited a $\mathrm{pH}$-responsive prolonged release property. In addition, the cellular uptake study demonstrated that FCPP-NEMs serve as an efficient carrier to enhance the delivery of the entrapped drug into the target lung tumor cells. Moreover, the GFB/FCPP-NEMs induced a superior cytotoxic effect compared with free gefitinib. The inhibitory concentration to achieve 50\% cell death ( $\mathrm{IC}_{50}$ ) of GFB/FCPP-NEMs in A549 cells was 3.82-fold lower than that of free gefitinib. According to these results, FCPP-NEMs hold a great potential as a multifunctional and high-performance biomaterial for lung tumor targeting delivery, $\mathrm{pH}$-responsive sustained release, facilitated cellular uptake, and enhanced antitumor effect of antitumor drugs, like gefitinib.
\end{abstract}

Keywords: nanocomposites; controlled delivery; dual-particulate; cellular uptake; carboxymethyl $\beta$-glucan; chemotherapeutic drugs

\section{Introduction}

According to the 2018 report of the World Health Organization (WHO), lung cancer, particularly non-small cell lung cancer (NSCLC), is the leading cause of cancer-related human death worldwide. Until now, depending on the cancer cell type and stage, lung cancer has been treated primarily by chemotherapy, radiotherapy, surgery or a combination of them [1,2]. However, due to the high toxicity of most anticancer drugs, undesirable tissue distribution, inadequate penetration, and rapid clearance from blood circulation, chemotherapy often has in low efficacy, severe systemic toxicity and adverse effects $[3,4]$.

Gefitinib is a tyrosine kinase inhibitor that targets the epidermal growth factor receptor (EGFR), which is overexpressed in lung, colon, breast, brain, and ovarian tumors. Due to the dramatic 
clinical response to gefitinib in NSCLC, gefitinib was approved by the United States (US) Food and Drug Administration (FDA) in 2015 as the first-line treatment for patients with NSCLC [3]. However, a high dose ( $250 \mathrm{mg} /$ day) of gefitinib is required for its commercial tablets due to its low aqueous solubility, high P-glycoprotein efflux, as well as first-pass metabolism $[5,6]$. Consequently, oral gefitinib treatment leads to dose-related adverse effects, such as gastrointestinal disorders, nutritional as well as metabolism imbalances, and even, interstitial lung disease, which is fatal in some patients [7]. All the above-mentioned issues indicate that there is a need to design a novel approach or more effective and safer formulation, for the delivery of gefitinib, preferably as a pulmonary inhalant. Pulmonary inhalant would target delivery of therapeutics to the lungs with effective drug concentrations, and afford rapid onset of therapeutic action as well as minimal systemic side effects [8].

Over the last decade, the biodegradable and body-friendly polymeric micro/nanoparticles (NPs) have played an important role in cancer treatment. Among the various polymeric materials, poly(lactic-co-glycolic acid) (PLGA), a pharmaceutical excipient in the US Pharmacopeia and National Formulary (USP/NF) monograph, has become very popular in drug delivery systems due to its modified particle sizes, stable solid structure, facile surface functionalization, and excellent biocompatibility and biodegradability $[9,10]$. As a result of their stable micro/nanoscale solid structure, PLGA NPs exhibit an excellent ability to control release of their incorporated drug, which may be of value to prolong the systemic circulation time. More importantly, surface modification of PLGA NPs with functional polymers can confer them additional favorable characteristics. It is well established that chitosan (CS)-coated PLGA NPs have enhanced muco-adhesiveness and enabled sustained delivery of peptides to lungs [11]. Recently, Vasudev et al. prepared trimethyl CS chloride- modified PLGA NPs and demonstrated that these NPs combine the controlled release property of PLGA NPs with the muco-adhesive property of trimethyl CS chloride for oral insulin delivery [12]. In addition, Dhas et al. used FA-conjugated CS-coated PLGA NPs for delivery of bicalutamide in prostate cancer [13]. Unlike the above-mentioned surface modification, some reports have described the incorporation of a functional polymer into PLGA NPs. For example, Zhu et al. used a co-matrix of PLGA and D-tocopherol polyethylene glycol (1000) succinate (TPGS) to control the release rate, enhance encapsulation efficiency, and the antitumor effect of docetaxel [14]. Together, these findings suggest that the combination of PLGA and functional polymers may be a suitable strategy to achieve efficient delivery of antitumor drugs.

Therefore, the design and fabrication of a novel FA-conjugated and CS-coated poloxamer 407 (P407)/PLGA NPs (FCCP NPs) co-matrix nanocarrier for gefitinib delivery is highly desirable. This hybridized nanocarrier, FCPP NPs, would combine the unique benefits of FA, CS, PLGA NPs, and P407 into a single carrier system. On the one hand, the FA-conjugated CS affords the FA receptor (overexpressed on the cell surface of many human epithelial tumor cells) targeted feature, mucoadhesive property and $\mathrm{pH}$-triggered release control capacity $[15,16]$. On the other hand, $\mathrm{P} 407$, as a co-matrix agent, can enhance drug permeability by reducing the P-glycoprotein efflux of tumor cell membranes, and thus suppresses the multidrug resistance of the tumor cells [17].

However, despite the multiple attractive advantages of FCPP NPs, nanoscale particles are not suitable for efficient pulmonary inhalation delivery $[8,18]$. To address this issue, it is necessary to encapsulate these FCPP NPs into soluble and biodegradable porous microcapsules (MCs, specifically 1-5 $\mu \mathrm{m}$ ) that can effectively deposit in the lungs, and then dissociate to their primary particles (FCPP NPs) for tumor-targeted delivery. Among the various available MC materials, carboxymethyl $\beta$ - glucan (a water-soluble $\beta$-glucan derivative) may be an excellent candidate due to its high biocompatibility, biodegradability, and bioadhesion [19]. More importantly, carboxymethyl $\beta$-glucan is a polysaccharide, which has well-known immunomodulatory properties, including direct activation of NK cells and cytokine release by specialized phagocytes that stimulate phagocytosis [20]. Quite recently, a new approach using resveratrol and carboxymethyl $\beta$-glucan has been developed to treat respiratory diseases [21]. Accordingly, these findings make it very tempting to undertake the fabrication of a multifunctional biocompatible and stable NEM system for lung- targeted antitumor drug delivery 
via the encapsulation of these FCPP NPs into carboxymethyl $\beta$-glucan MCs, which, to the best of our knowledge, has not been reported to date.

Therefore, in this study, we sought to develop such a novel type of FCPP-NEM carrier system to achieve effective lung tumor-targeted delivery, $\mathrm{pH}$-responsive sustained release, facilitated cellular uptake, and enhanced antitumor effects of the antitumor drugs, like gefitinib. To achieve this goal, we systematically evaluated the physicochemical properties of the newly developed GFB/FCPPNEMs, including morphology, particle size distribution (PSD), crystalline state, drug loading amount, Fourier transform infrared (FT-IR) spectrum, drug release profiles, and release mechanism. In addition, besides studying the cellular uptake of FCPP NPs (the primary particles of FCPP-NEMs), the cytotoxicity of GFB/FCPP-NEMs was evaluated in the lung cancer cell lines A549 and PC-9. Additionally, an accelerated stability study was conducted to determine the physicochemical stability of GFB/FCPP-NEMs.

\section{Materials and Methods}

\subsection{Materials}

P407 was obtained from BASF SE (Ludwigshafen, Germany). Carboxymethyl $\beta$-glucan was purchased from Yuancheng Chemical Co., Ltd. (Suzhou, China). PLGA (Mw = 24,000-38,000, lactide/glicolide $=50 / 50$ ) was provided by Daigang Biomaterials (Jinan, China). USP grade gefitinib (purity $\geq 99 \%$ ) was obtained from Yadong Pharma Ltd. (Nanjing, China). FA, polyvinyl alcohol (PVA), and CS ( $\mathrm{Mw}=8000 \mathrm{Da}$, degree of deacetylation $\geq 75 \%$ ) were purchased from Aladdin Reagent Co., Ltd. (Shanghai, China). High-performance liquid chromatography (HPLC) grade acetonitrile and methanol were purchased from Sinopharm Chemical Reagent Co., Ltd. (Shanghai, China). The other chemicals and reagents were of analytical grade.

\subsection{Cell Culture}

The human NSCLC cell lines A549 and PC-9 were obtained from the Chinese Academy of Sciences (Shanghai, China). A549 and PC-9 cells were cultured in high glucose Dulbecco's modified Eagle's medium (DMEM) and Roswell Park Memorial Institute (RPMI)-1640 medium, respectively. Both culture media were supplemented with antibiotic (100 U/mL penicillin and $100 \mu \mathrm{g} / \mathrm{mL}$ streptomycin) and $10 \%$ fetal bovine serum. The cells were cultured under humidified conditions at $37^{\circ} \mathrm{C}$ and $5 \% \mathrm{CO}_{2}$.

\subsection{Fabrication of GFB/FCPP NPS}

The monodispersed GFB/FCPP NPs were prepared by a two-step process: First, the gefitinibloaded P407/PLGA (GFB/P407/PLGA) NPs were fabricated by a double emulsion-solvent evaporation method (Step 1 in Figure 1) [22]. Briefly, $20 \mathrm{mg}$ of P407, $10 \mathrm{mg}$ of gefitinib, and $80 \mathrm{mg}$ of PLGA were dissolved in $7 \mathrm{~mL}$ of dichloromethane (DCM) to obtain the organic phase. Subsequently, $2 \mathrm{~mL}$ of distilled water, used as the aqueous phase, was poured into the above organic phase, and mixed by stirring at 12,000 rpm for $6 \mathrm{~min}$ to obtain a coarse emulsion. Then, the coarse emulsion was sonicated with an ultrasonic probe for $6 \mathrm{~min}$ (pulsing mode $3 \mathrm{~s}$ on and $2 \mathrm{~s}$ off, $300 \mathrm{Walt}$ ) in an ice water bath. Next, the resulting emulsion was poured into $30 \mathrm{~mL}$ of a $1 \%$ PVA aqueous solution and the mixture was further sonicated for $6 \mathrm{~min}$ in an ice water bath. After sonication, the resulting double emulsion was stirred overnight using a propeller-type agitator at $200 \mathrm{rpm}$ to remove the DCM. Afterwards, the suspension was centrifugated at $9000 \mathrm{rpm}$ for $15 \mathrm{~min}$, washed with distilled water and the GFB/P407/PLGA NPs were collected.

In the subsequent step, the obtained GFB/P407/PLGA NPs were coated with FA-conjugated CS and then lyophilized to form the GFB/FCPP NPs (Step 2 in Figure 1). Briefly, FA conjugated CS was prepared based on a carbodiimide reaction as previously reported (Supplementary Materials) [23]. Then, $0.5 \mathrm{~g}$ of GFB/P407/PLGA NPs was resuspended in $10 \mathrm{~mL}$ of the FA-conjugated CS solution ( $1 \%$, in $1 \%$ acetic acid solution). Next, the resulting mixture was vortexed for 5 min and stirred for 
$3 \mathrm{~h}$. After centrifugation at $9000 \mathrm{rpm}$ for $20 \mathrm{~min}$, the collected GFB/FCPP NPs were washed with distilled water, and lyophilized at $-55{ }^{\circ} \mathrm{C}$ for $10 \mathrm{~h}$ using a laboratory-scale freeze-drying device (Evela Instruments, Tokyo, Japan) at a pressure below $10 \mathrm{~Pa}$. Ultimately, the lyophilized powder was passed through a 80-mesh sieve, and stored in a screw cap sealed glass bottle.

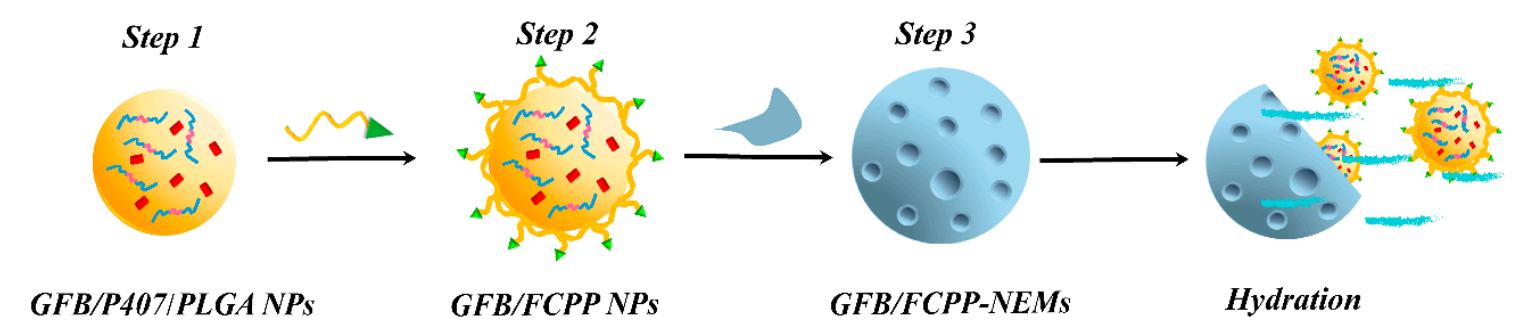

PLGA

Gefitinib

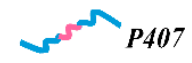

$\sim F A-C S$

Carboxymethyl $\beta$-glucan

Figure 1. Schematic diagram of the preparation of gefitinib-loaded and folic acid (FA)-conjugated chitosan (CS)-coated poloxamer 407 (P407)/poly (lactic-co-glycolic acid) (PLGA) nanoparticles (NPs), namely GFB/FCPP NPs and GFB/FCPP NPs embedded in microcapsules (MCs), namely GFB/FCPP- NEMs.

\subsection{Fabrication of GFB/FCPP-NEMs}

The encapsulation of GFB/FCPP NPs in carboxymethyl $\beta$-glucan MCs was carried out by the well-known spray drying method (Step 3 in Figure 1). In a typical preparation, $1 \mathrm{~g}$ of carboxymethyl $\beta$-glucan was dissolved in $100 \mathrm{~mL}$ of purified water to form a $1 \%$ carboxymethyl $\beta$-glucan aqueous solution. Then, $0.4 \mathrm{~g}$ GFB/FCPP NPs was dispersed in the above solution under magnetic stirring until a uniform dispersion was formed. Eventually, the dispersion was pumped into an ADL-310 mini bench-top spray dryer (Nanjing, China) to obtain the GFB/FCPP-NEMs. The inlet and outlet temperatures of the drying chamber were set to 170 and $60^{\circ} \mathrm{C}$, respectively. The atomization pressure was set to $170 \mathrm{kPa}$. The drying air flow rate and feed rate were $0.6 \mathrm{~m}^{3} / \mathrm{min}$ and $5.0 \mathrm{~mL} / \mathrm{min}$, respectively. The GFB/FCPP NPs dispersion was continuously stirred during the drying process. Finally, the resulting dry powder was stored in a screw cap sealed glass bottle.

\subsection{Characterization of the GFB/FCPP NPS and GFB/FCPP-NEMs}

\subsubsection{Morphological Analysis}

Particle shape and surface morphology of the powdered GFB/P407/PLGA NPs, GFB/FCPP NPs, and GFB/FCPP-NEMs were examined by scanning electron microscopy (SEM) using a Teneo VS ${ }^{\mathrm{TM}}$ scanning electron microscope (FEI Co., Hillsboro, OR, USA). Before SEM analysis, a small section of each sample was sputtered with a thin gold/palladium layer for conductivity.

\subsubsection{Particle Size Distribution and Zeta Potential Measurements}

For the prepared GFB/P407/PLGA NPs and GFB/FCPP NPs, the mean hydrodynamic diameter, polydispersity index, and surface charge were measured using a Nicomp ${ }^{\mathrm{TM}} 380$ ZLS zeta potential/size analyzer (Particle Sizing Systems, Santa Barbara, CA, USA). Prior to measurements, each sample was appropriately diluted and highly dispersed in distilled water. For the prepared GFB/FCPP-NEMs powder, laser diffraction was used to determine the particle size distribution using a WJL-612 laser diffraction particle size analyzer (Rise Instruments Co., Ltd., Nanjing, China). The particle size was expressed as the median diameter $\left(d_{50}\right)$. The span values $=\left[d_{90}-d_{10}\right] / d_{50}$, where $d_{90}, d_{50}$ and $d_{10}$ indicate the equivalent volume diameters corresponding to the $90 \%, 50 \%$, and $10 \%$ points of the cumulative distribution curve, respectively. All the measurements were performed in triplicate for each sample. 


\subsubsection{Solid-State Test}

The thermal behavior of crude gefitinib, GFB/P407/PLGA NPs, physical mixture of gefitinib and P407/PLGA NPs, GFB/FCPP NPs, carboxymethyl $\beta$-glucan, and GFB/FCPP-NEMs was analyzed by differential scanning calorimetry (DSC) using an NY-101B, differential scanning calorimeter (Netzyu Electronics Technology Co., Ltd., Shanghai, China). Powder samples (approximately 10-16 mg) were accurately weighed, placed in ceramic crucibles with a lid under a constant purge of nitrogen at $40 \mathrm{~mL} / \mathrm{min}$. Each sample was scanned from 40 to $300^{\circ} \mathrm{C}$ (at a ramp rate of $10^{\circ} \mathrm{C} / \mathrm{min}$ ).

\subsubsection{FT-IR Spectroscopy Analysis}

FT-IR spectra of the FA conjugated CS, crude gefitinib, P407/PLGA NPs, physical mixture of gefitinib and P407/PLGA NPs, GFB/FCPP NPs, and GFB/FCPP-NEMs were recorded using a Bruker IFS 55 FT-IR spectrometer (Bruker Optics GmbH, Ettlingen, Germany). Before measurement, each sample was ground and pressed into potassium bromide discs.

\subsubsection{Drug Loading Amount Measurements}

Gefitinib content was determined by HPLC analysis using the Agilent 1200 series HPLC system (Agilent Technologies, Santa Clara, CA, USA), which consisted of a tunable UV detector, an autosampler, and a column heater (set at $35^{\circ} \mathrm{C}$ ). The stationary phase was a reverse phase Agilent Zorbax Eclipse Plus C18 column, while the mobile phase was a mixture of acetonitrile and $10 \mathrm{mM}$ ammonium dihydrogen phosphate buffer $(51: 49, v / v)$. To determine the drug loading amount and entrapment efficiency (EE) of each gefitinib formulation, a predetermined amount of GFB/FCPP NPs or GFB/FCPP-NEMs powder was added in $4 \mathrm{~mL}$ of a mixture solution (DCM: acetonitrile, 50/50), and continuously vortexed for $10 \mathrm{~min}$ to ensure complete release of the encapsulated gefitinib. Subsequently, the solvent was evaporated under nitrogen. Then, $4 \mathrm{~mL}$ of acetonitrile was added to the extract and vortex mixed for $2 \mathrm{~min}$ followed by agitation overnight to dissolve the gefitinib. After centrifugation at $9000 \mathrm{rpm}$ for $5 \mathrm{~min}$, the supernatant was collected for HPLC analysis. The EE was defined as: the amount of gefitinib in the samples $\times 100 \%$ /total amount of gefitinib added during the fabrication of the samples. The drug loading amount was expressed as: total amount of gefitinib in the samples $\times 100 \% /$ the amount of the samples.

\subsubsection{In Vitro Drug Release Test}

A dialysis bag diffusion method was used to examine the drug release behaviors of GFB/FCPP NPs, and GFB/FCPP-NEMs under two different $\mathrm{pH}$ values. Briefly, raw gefitinib, GFB/FCPP NPs, or GFB/FCPP-NEMs with equal amount of gefitinib $(4 \mathrm{mg})$ were individually suspended in $2 \mathrm{~mL}$ of phosphate-buffered solution (PBS, pH 7.4) containing 1\% Tween 80 and sealed in a pre-swelled cellulose acetate dialysis bag. Then, the dialysis bag was immersed in another $100 \mathrm{~mL}$ of PBS and shaken horizontally at $100 \mathrm{rpm}$ and $37^{\circ} \mathrm{C}$ for $48 \mathrm{~h}$. Tween 80 was used to maintain the sink conditions. At the scheduled time intervals, samples of approximately $4.0 \mathrm{~mL}$ were collected from the release medium and replaced with fresh PBS. Each sample was filtered (through a $0.45-\mu \mathrm{m}$ membrane), transferred into a vial and the released amount of drug was determined by HPLC analysis. Additionally, to evaluate the $\mathrm{pH}$-responsive controlled release property of GFB/FCPP NPs and GFB/FCPP-NEMs, the above-mentioned analysis was performed with pH 5.0 PBS as another release medium. Different kinetic models of zero-order, first-order, Higuchi, and Korsmeyer-Peppas were used to investigate potential release mechanisms of GFB/FCPP-NEMs [24].

\subsection{Cellular Uptake Study}

The primary particles of FCPP-NEMs, namely FCPP NPs, taken up by A549 cells were determined by fluorescence microscopy analysis. Coumarin- 6 was used to visualize FCPP NPs. The preparation method for coumarin-6-loaded FCPP NPs was the same as that used for GFB/FCPP NPs except that gefitinib was 
replaced with coumarin-6. For cellular uptake experiments, A549 cells were seeded on cover slips at a density of $1 \times 10^{5}$ cells/well in 12-well plates and incubated for $24 \mathrm{~h}$. After removal of the culture medium, A549 cells were incubated with $1 \mathrm{~mL}$ DMEM containing coumarin- 6-loaded FCPP or coumarin-6-loaded P407/PLGA samples. Following incubation for $4 \mathrm{~h}$, the A549 cells on the cover slips were washed with PBS, fixed with $4 \%$ paraformaldehyde for $10 \mathrm{~min}$ at room temperature, and then washed again with PBS. After staining with Hoechst 33,342, the A549 cells were washed with PBS. Then, the cover slip containing A549 cells was covered with 50\% glycerol and visualized by fluorescence microscopy using an Olympus IX73 fluorescence microscope (Olympus Corp., Tokyo, Japan).

\subsection{Cytotoxicity Evaluation of GFB/FCPP-NEMs}

The in vitro anticancer activity of GFB/FCPP-NEMs was evaluated in two lung cancer cell lines (A549 and PC-9 cells) by the colorimetric MTT (3-(4,5-dimethylthiazol-2-yl)-2,5-diphenyltetrazolium bromide) assay. Briefly, A549 and PC-9 cells were seeded in 96-well plates at the density of $1 \times 10^{4}$ cells/well in $100 \mu \mathrm{L}$ of normal culture medium, and cultured overnight. Afterwards, the culture medium was replaced with $100 \mu \mathrm{L}$ of DMEM or RPMI-1640 containing serial concentrations of gefitinib or GFB/FCPP-NEMs. The graded concentration of gefitinib ranged from 0.2 to $20 \mu \mathrm{g} / \mathrm{mL}$. After incubation at $37^{\circ} \mathrm{C}$ for $48 \mathrm{~h}$, the treatment medium was aspirated from the wells, and replaced with $100 \mu \mathrm{L}$ of fresh culture medium containing the MTT solution $(20 \mu \mathrm{L}, 5 \mathrm{mg} / \mathrm{mL})$. After incubation for $4 \mathrm{~h}$, the MTT solution was aspirated, and the formed formazan crystals were solubilized with $100 \mu \mathrm{L}$ of dimethyl sulfoxide. Next, the absorbance of the resulting solution was detected at $490 \mathrm{~nm}$ using a microplate reader (BioTek, Winooski, VT, USA). Cells treated with normal medium served as the control cells. The cell viability was calculated as follows: optical density (OD) of test cells $\times 100 \% / O D$ of control cells. Additionally, empty FCPP-NEMs were also tested by this procedure.

\subsection{Physicochemical Stability}

To evaluate the physicochemical stability of GFB/FCPP-NEMs, an accelerated stability analysis was performed under the accelerated conditions currently recommended by the International Conference on Harmonization guideline. The GFB/FCPP-NEMs were stored in a screw cap sealed glass bottles in a humidity chamber $(75 \% \mathrm{RH})$ at constant temperature $\left(40{ }^{\circ} \mathrm{C}\right)$. At the specified time points $(1,2$, and 3 months), their particle size, physical state, drug content and related substances of gefitinib were determined.

\subsection{Statistical Analysis}

All results were expressed as the mean \pm standard deviation (SD) of three independent experiments. All statistical analyses were performed using the SPSS, predictive analysis software (PASW), Statistic Software (SPSS/IBM Corp., Armonk, NY, USA). Comparison between groups was performed by Student's $t$-tests. $P$-values $<0.05$ were considered statistically significant [15].

\section{Results and Discussion}

\subsection{Preparation and Characterization of GFB/FCPP NPS and GFB/FCPP-NEMs}

The processes for the fabrication of monodispersed GFB/FCPP NPs and GFB/FCPP-NEMs are depicted in Figure 1. In the first process, the GFB/FCPP NPs, which serve as nanoscale drug reservoirs, were prepared by an emulsion-diffusion solvent evaporation process followed by coating with FA-conjugated CS. As shown in Figure S1, the gefitinib crystals consisted of irregularly shaped and dozens of micrometer-sized particles. Unlike gefitinib crystals, the prepared GFB/P407/PLGA samples consisted of a large number of regular spherical NPs with smooth surface without any noticeable cracks (Figure 2A). Moreover, hardly any gefitinib crystals was visible in the GFB/P407/PLGA sample, suggesting that most gefitinib molecules were encapsulated in the P407/PLGA NPs, which was critical for attaining sustained deliver, enhanced cellular uptake, and thereby improving the cytotoxicity of the 
encapsulated gefitinib molecules $[9,24,25]$. As shown in Figure 2B, after the FA-conjugated CS coating (Step 2 in Figure 1), the degree of sphericity of the GFB/FCPP NPs was reduced and their surface was relatively coarse compared with that of the GFB/P407/PLGA NPs. The hydrodynamic diameter, surface charge and drug loading amount of the prepared NPs are listed in Table 1. Based on the dynamic light scattering results, the GFB/FCPP NPs had a mean size of $255.4 \pm 14.5 \mathrm{~nm}$ with a low polydispersity index, suggesting that the GFB/FCPP NPs were monodispersed. The drug loading amount and EE for GFB/FCPP NPs were $7.68 \pm 0.36 \%$ and $95.57 \pm 1.33 \%$, respectively. GFB/FCPP NPs exhibited nanoscale sizes and a narrow size distribution, but nanoscale particles are not suitable for efficient pulmonary delivery. In order to achieve an effective deposition of the gefitinib formulation in deep lung regions (with huge inner surface area), the diameter of the powder particles is required to range from 1 to 5 $\mu \mathrm{m}$ [18]. Thus, the biodegradable and biocompatible polymer excipient, carboxymethyl $\beta$-glucan, was used as the MC material and the commonly used spray-drying technique was used for the encapsulation of GFB/FCPP NPs in carboxymethyl $\beta$-glucan MCs (Step 3 in Figure 1). Remarkably, as shown in Figure 2C, the formed GFB/FCPP-NEMs were nearly spherical capsules with a highly porous and sunken surface (their pore size was around $20 \mathrm{~nm}$, inset of Figure $2 \mathrm{C}$ ). The median diameter $\left(\mathrm{d}_{50}\right), \mathrm{d}_{10}$, $\mathrm{d}_{90}$ and span of GFB/FCPP-NEMs were about $2.2 \pm 0.1 \mu \mathrm{m}, 1.2 \pm 0.1 \mu \mathrm{m}, 4.6 \pm 0.3 \mu \mathrm{m}$ and $1.6 \pm 0.2 \mu \mathrm{m}$, respectively. Due to their highly porous surface, GFB/FCPP-NEMs can quickly dissociate into its primary particles (GFB/FCPP NPs) for FA-mediated tumor-targeted drug delivery after hydration, as shown in Figure 2D.

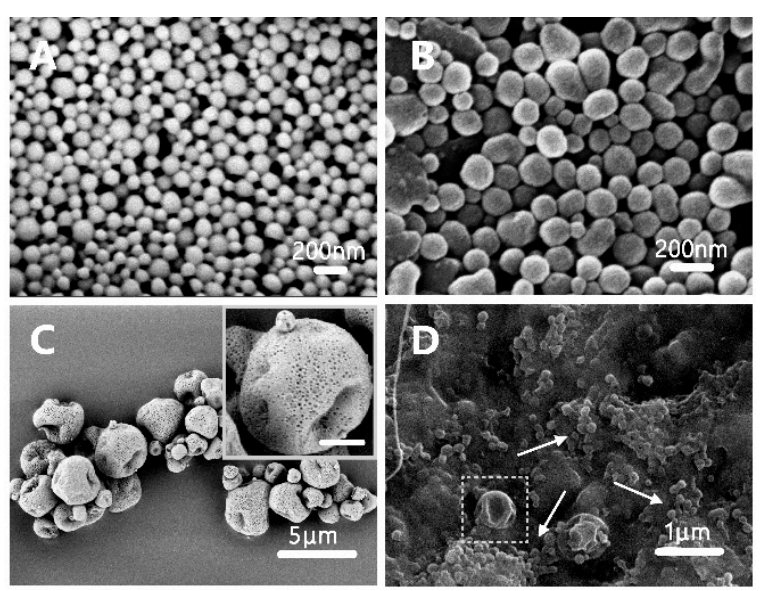

Figure 2. Scanning electron microscopy (SEM) photomicrographs of (A) GFB/P407/PLGA NPs, (B) GFB/FCPP NPs, (C) GFB/FCPP-NEMs (inset, high magnification image, scale bar $=1 \mu \mathrm{m}$ ), and (D) GFB/FCPP-NEMs after exposure to PBS (pH 7.4). The arrow is pointing to the GFB/FCPP NPs, while the square frame contains a GFB/FCPP-NEM.

Table 1. Characterization of different samples $(n=3)$.

\begin{tabular}{cccccc}
\hline Formulations & $\begin{array}{c}\text { Particle } \\
\text { Size }(\mathbf{n m})\end{array}$ & $\begin{array}{c}\text { Polydispersity } \\
\text { Index }\end{array}$ & $\begin{array}{c}\text { Zeta Potential } \\
\mathbf{( m V )}\end{array}$ & 1 EE (\%) $^{\mathbf{1} \text { ) }}$ & $\begin{array}{c}\text { Drug } \\
\text { Loading (\%) }\end{array}$ \\
\hline P407/PLGA NPs & $175.8 \pm 11.2$ & $0.08 \pm 0.01$ & $-11.74 \pm 1.24$ & - & - \\
GFB/P407/PLGA NPs & $180.0 \pm 12.3$ & $0.16 \pm 0.05$ & $-12.54 \pm 2.30$ & $90.42 \pm 0.89$ & $12.96 \pm 0.41$ \\
GFB/FCPP NPs & $255.4 \pm 14.5$ & $0.24 \pm 0.09$ & $+3.75 \pm 1.85$ & $95.57 \pm 1.33$ & $7.68 \pm 0.36$ \\
\hline
\end{tabular}

${ }^{1} \mathrm{EE}$, entrapment efficiency.

\subsection{Solid-State Characterization}

Thermal analysis has been widely used for determination of the physical state of drugs in the NPs or MCs. Thus, DSC analysis of pure gefitinib, GFB/P407/PLGA, the physical mixture of gefitinib and P407/PLGA NPs, GFB/FCPP NPs, and GFB/FCPP-NEMs was conducted and the obtained DSC 
thermograms are presented in Figure 3. As shown in Figure 3a, pure gefitinib crystals show an endothermic peak at around $195^{\circ} \mathrm{C}$ (with an enthalpy of $390 \mathrm{~J} / \mathrm{g}$ ), which corresponds to its melting temperature. The DSC curve of the physical mixture (gefitinib and P407/PLGA NPs) shown in Figure $3 \mathrm{~b}$ also exhibited the sharp peak associated with gefitinib, indicating that gefitinib was still present in a crystalline state. However, after loading gefitinib into P407/PLGA NPs, the endothermic peak of gefitinib is not observed in the DSC thermograms of GFB/P407/PLGA NPs (Figure 3c). The absence of the endothermic peak corresponding to crystalline gefitinib in the thermograms indicated that the gefitinib entrapped in the P407/PLGA NPs had mostly lost its crystallinity [26]. Similarly, the endothermic peak of crystalline gefitinib also disappeared for both the GFB/FCPP NPs and GFB/FCPP-NEMs samples (Figure 3e,g). According to the DSC measurements, the encapsulated gefitinib was mostly in an amorphous state in the GFB/FCPP-NEMs delivery system.

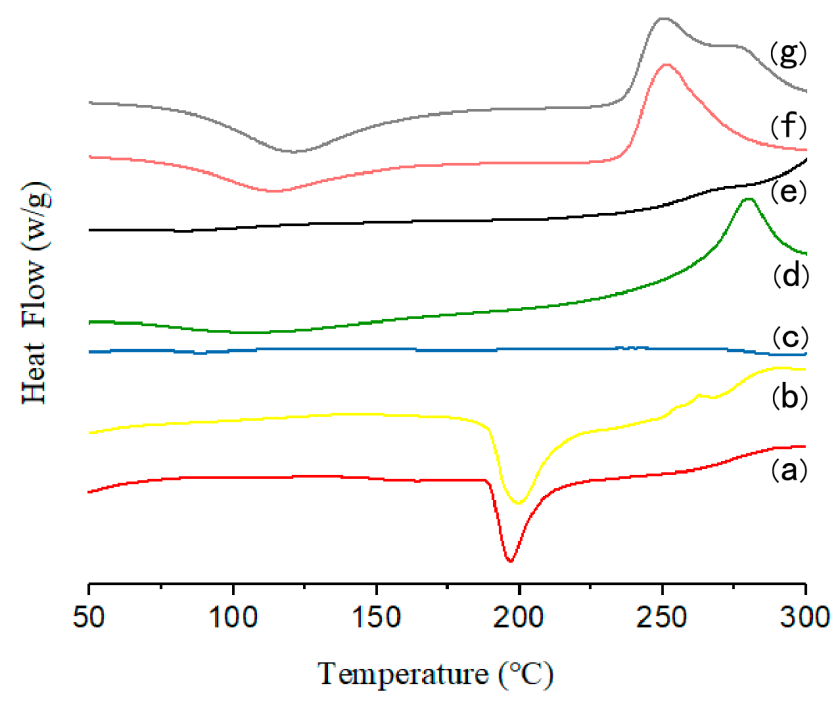

Figure 3. Differential scanning calorimetry (DSC) thermograms of (a) gefitinib crystals, (b) physical mixture of gefitinib and P407/PLGA NPs, (c) GFB/P407/PLGA NPs, (d) FA-conjugated CS, (e) GFB/FCPP NPs, (f) carboxymethyl $\beta$-glucan, and (g) GFB/FCPP-NEMs.

\subsection{FT-IR Characterization}

The FT-IR spectra of CS, FA, and FA-conjugated CS samples are displayed in Figure S2. For the FA sample, the typical band at $1694 \mathrm{~cm}^{-1}$ is attributed to the $\mathrm{C}=\mathrm{O}$ stretching vibration, while the band at $1606 \mathrm{~cm}^{-1}$ is the characteristic peak of the amino group in the pteridine ring. In addition, the peak at $1484 \mathrm{~cm}^{-1}$ corresponds to the vibration of the benzene ring [23]. For the CS sample, the characteristic absorption peaks around 1619 and $1092 \mathrm{~cm}^{-1}$ belong to the $\mathrm{N}-\mathrm{H}$ and $\mathrm{C}-\mathrm{O}$ stretching vibrations, respectively. After conjugation of FA with CS, the absorption peak at $1514 \mathrm{~cm}^{-1}$ was weakened as a result of the reaction of the amino group of CS with the carboxylate group of FA. In addition, the appearance of a new peak at $1606 \mathrm{~cm}^{-1}$, which corresponds to the amino group in the pteridine ring of FA, also confirmed that FA was conjugated with CS.

The FT-IR spectra of pure gefitinib, GFB/P407/PLGA, the physical mixture of gefitinib and P407/PLGA NPs, GFB/FCPP NPs, and GFB/FCPP-NEMs are presented in Figure 4. Pure gefitinib displays several absorption peaks. The FT-IR spectrum of gefitinib shown in Figure 4a reveals a characteristic absorption peak at $1625 \mathrm{~cm}^{-1}$ that was attributed to $\mathrm{C}=\mathrm{N}$ stretching vibrations, while the peaks at $1110 \mathrm{~cm}^{-1}$ and $1028 \mathrm{~cm}^{-1}$ corresponded to the $\mathrm{C}-\mathrm{O}$ and $\mathrm{C}-\mathrm{F}$ stretching vibrations, respectively [27]. As anticipated, these gefitinib absorption peaks are also present in the spectrum of the physical mixture of gefitinib and P407/PLGA NPs (Figure 4c). Compared with the physical mixture, most of the gefitinib characteristic absorption bands were weakened in the FT-IR spectrum of the GFB/P407/PLGA sample (Figure 4d), suggesting that gefitinib was entrapped in the P407/PLGA NPs. 
After FA-conjugated CS coating, the absorption peaks at 1694, 1606, and $1484 \mathrm{~cm}^{-1}$ corresponding to the FA-conjugated CS can be observed in the FTIR spectrum of the GFB/FCPP NPs (Figure 4e). Moreover, the absorption peaks of GFB/FCPP-NEMs (Figure 4g) were similar to those of the blank carboxymethyl $\beta$-glucan MCs, except for the intense peak at $1752 \mathrm{~cm}^{-1}$ corresponding to the $\mathrm{C}=\mathrm{O}$ bond of the ester group from PLGA. Combine with the SEM analysis, these results clearly suggest that GFB/FCPP NPs and GFB/FCPP-NEMs were successfully prepared.

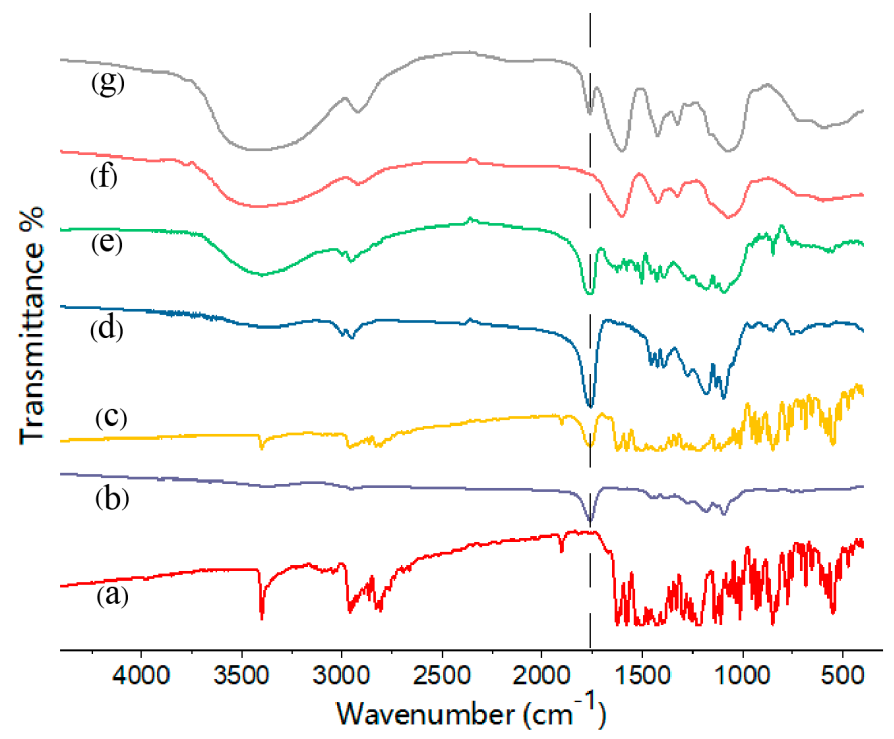

Figure 4. Fourier transform infrared (FT-IR) spectra of (a) gefitinib crystals, (b) P407/PLGA NPs, (c) physical mixture of gefitinib and P407/PLGA NPs, (d) GFB/P407/PLGA NPs, (e) GFB/FCPP NPs, (f) carboxymethyl $\beta$-glucan, and (g) GFB/FCPP-NEMs.

\subsection{Drug Release Profiles}

In vitro drug release tests were conducted at two different $\mathrm{pH}$ values $(\mathrm{pH} 7.4$ and $\mathrm{pH} 5.0$ ) to simulate the systemic circulation conditions and weakly acidic environments inside the tumor sites, respectively. The results revealed that the release rate of gefitinib from the gefitinib suspension was fast in both media. Even at pH 7.4 (mimic physiological conditions), approximately $60 \%$ of gefitinib was released from the gefitinib suspension within $4 \mathrm{~h}$ (Figure 5A). Unlike the rapid release pattern of the gefitinib suspension, the release profiles of gefitinib from GFB/FCPP NPs exhibited a slow and prolonged drug release behavior. Only about $13 \%$ of gefitinib was released in the initial $4 \mathrm{~h}$, and the cumulative release rate of gefitinib from GFB/FCPP NPs was no more than $60 \%$ after 48 h. Compared with gefitinib suspension, the sustained-release behavior of GFB/FCPP NPs can be attributed to the encapsulation of gefitinib into FCPP NPs, which can lessen the damage of gefitinib to normal cells in the human body $[28,29]$. In addition, it is worth noting that the release of gefitinib from GFB/FCPP- NEMs was slower than that of GFB/FCPP NPs. As shown in Figure S3, after hydration of carboxymethyl $\beta$-glucan MCs, a hydrophilic gel of carboxymethyl $\beta$-glucan was formed and surrounded the GFB/FCPP NPs, which contributed to the decreased release rate of gefitinib from GFB/FCPP-NEMs. As expected, the release rate of gefitinib from the GFB/FCPP NPs or GFB/FCPPNEMs at $\mathrm{pH} 5.0$ was significantly fast compared with that at $\mathrm{pH} 7.4$, as shown in Figure 5B. For the GFB/FCPP NPs, the amount of released gefitinib from at the sampling time of $2 \mathrm{~h}$ reached approximately $45 \%$ and was almost $60 \%$ within $4 \mathrm{~h}$ at $\mathrm{pH}$ 5.0. In addition, nearly $70 \%$ of gefitinib was released after $48 \mathrm{~h}$. The enhanced drug release rate of GFB/FCPP NPs can be attributed to the $\mathrm{pH}$-dependent FA-conjugated CS coating. As is well known, CS can be dissolved under acidic conditions. Since tumor microenvironment is weakly acidic, with $\mathrm{pH}$ values down to 5.0, the enhanced drug release efficiency of GFB/FCPP NPs at acidic $\mathrm{pH}$ would be beneficial in improving the cytotoxic and antitumor effects of 
the entrapped drug [30,31]. More importantly, GFB/FCPP NPs can release many more drug molecules at tumor sites than at normal tissues and thereby avoid side effects due to universal cytotoxicity. The above results indicated that the prepared FCPP-NEMs are likely an ideal drug delivery system for pH-dependent sustained drug release. Additionally, the probable release mechanism was investigated using different kinetic models of zero-order, first- order, Higuchi, and Korsmeyer-Peppas to fit the drug release curves of GFB/FCPP NPs and GFB/FCPP-NEMs, and their model parameters are summarized in Tables S1 and S2. The results revealed that the gefitinib release from both GFB/FCPP NPS and GFB/FCPP-NEMs was best fitted with the first-order model (with the highest value of regression coefficients, $r^{2} \geq 0.96$ ) suggesting diffusion-controlled drug release kinetics. In addition, based on the Korsmeyer-Peppas model, the release exponent ( $n$ ) for the tested GFB/FCPP NPs (at pH 5.0 and 7.4) or GFB/FCPP-NEMs (at pH 5.0) below 0.45, suggesting that the release of gefitinib from the test samples mainly follows the Fickian diffusion mechanism [32,33]. In contrast, the release exponent $(n)$ for the tested GFB/FCPP-NEMs at pH 7.4 up to 0.69 , indicated that gefitinib release from GFB/FCPP-NEMs was based on a combined diffusion/erosion process [34].

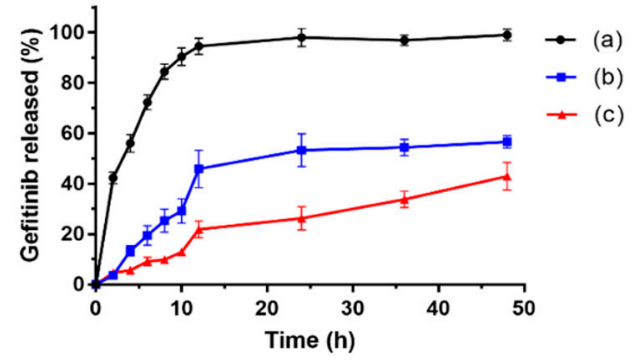

(A)

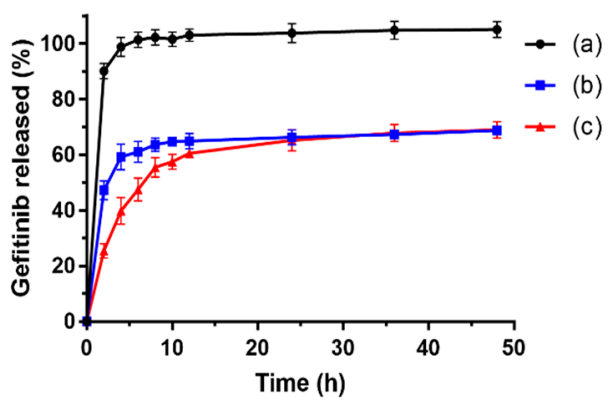

(B)

Figure 5. Drug release of different gefitinib formulations at (A) $\mathrm{pH} 7.4$ and (B) $\mathrm{pH}$ 5.0. (a) gefitinib suspension, (b) GFB/FCPP NPs, and (c) GFB/FCPP-NEMs. Each data point represents the mean \pm SD $(n=3)$.

\subsection{Cell Uptake and Intracellular Localization}

To confirm the cell uptake of the primary particles of FCPP-NEMs, namely FCPP NPs, a fluorescence imaging analysis was carried out. The fluorescence microscopy images of A549 cells after $4 \mathrm{~h}$ incubation with coumarin-6-loaded FCPP NPs are shown in Figure 6. For comparison purposes, the cellular uptake behavior of coumarin-6-loaded P407/PLGA NPs was also studied. As shown in Figure 6A,B, the nucleus of the cells was stained blue with the Hoechst 33,342 dye. Also, obvious green fluorescence can be observed in the cytoplasmic region of cells with both coumarin- 6- loaded NPs tested, indicating that both coumarin-6-loaded FCPP NPs and coumarin- 6- loaded P407/PLGA NPs can be internalized by the A549 cells. Moreover, a significant enhancement in the green fluorescence intensity in the cytoplasmic region was observed with the increase of the dose of each carrier, suggesting that the uptake of both carriers was dose-dependent. As anticipated, compared with coumarin-6-loaded P407/PLGA NPs, cells exposed to coumarin- 6- loaded FCPP NPs had a slightly stronger green fluorescence at concentrations from 50 to $500 \mu \mathrm{g} / \mathrm{mL}$. Such enhanced uptake can be attributed to the introduction of FA on the surface of the FCPP NPs. As is well known, FA has a high binding affinity for the FA receptor which is frequently overexpressed in human cancer cells. Several studies in the literature confirm that FA modified NPs can improve the chemotherapeutic efficacy of drugs due to their efficient cellular internalization through FA-mediated endocytosis [35]. Together, the results of the cell uptake study indicated that FCPP-NEMs can serve as an efficient carrier for enhanced delivery of a drug to tumor cells, thereby improving the antitumor effects of the encapsulated chemotherapeutic drug. 


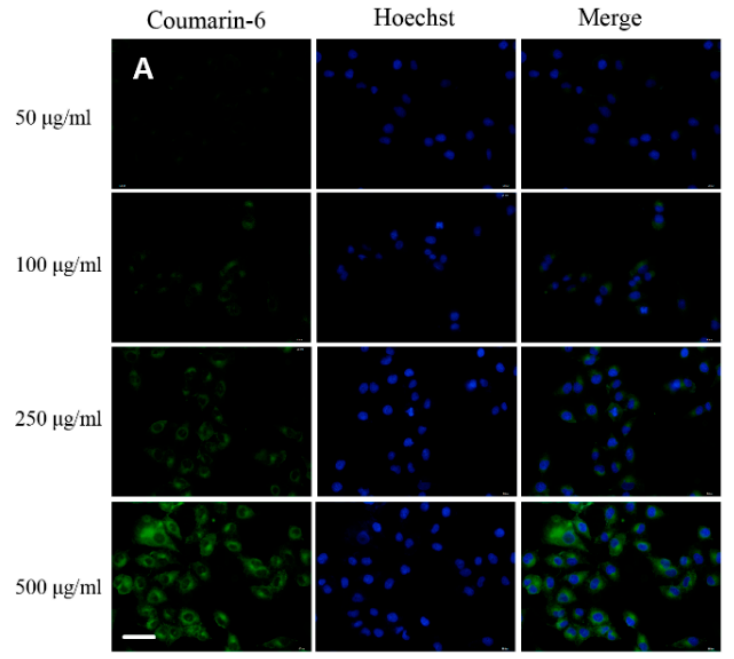

(A)

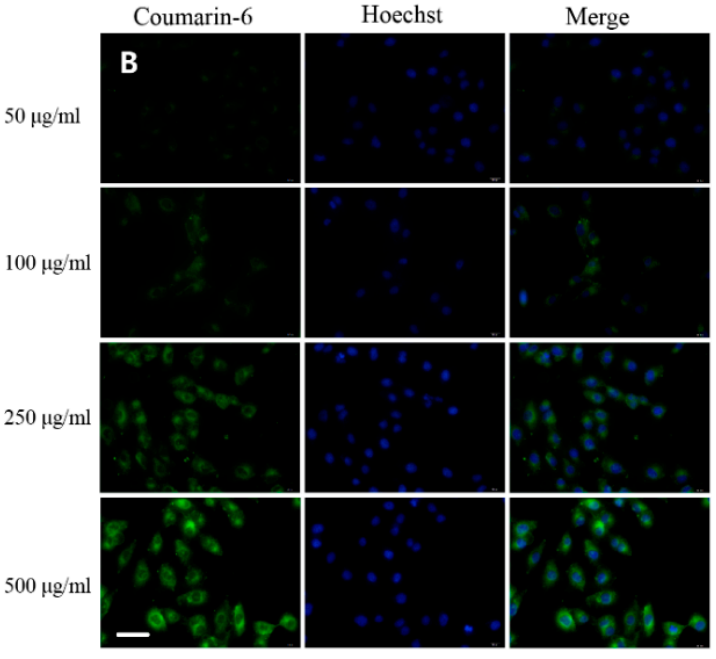

(B)

Figure 6. Fluorescence images of A549 cells after incubation with coumarin-6-loaded P407/PLGA NPs

(A) and coumarin-6-loaded FCPP NPs (B). Scale bar $=20 \mu \mathrm{m}$.

\subsection{Cytotoxicity}

After the drug release and cell uptake evaluation, the GFB/FCPP-NEMs may be assumed to display a more enhanced cytotoxic effect than gefitinib crystals. In order to confirm such an assumption, A549 and PC-9 cells were treated with blank FCPP-NEMs, gefitinib crystals, and GFB/FCPP-NEMs at various concentrations for $48 \mathrm{~h}$, and their cytotoxicity was assessed by the MTT colorimetric assay. The results presented in Figure 7 reveal that blank FCPP-NEMs had no apparent inhibitory effect on the proliferation of both A549 and PC-9 cells at the tested concentrations. More than $90 \%$ of the cells were viable at concentrations as high as $1000 \mu \mathrm{g} / \mathrm{mL}$, which indicated that the FCPP-NEMs had little cytotoxic effect on both A549 and PC-9 cells. Since CS, P407, PLGA and $\beta$-glucan are FDA-approved biodegradable and biocompatible polymers, FCPP-NEMs could be safely used as a drug delivery system for targeted treatment of lung tumors with chemotherapeutic agents. On the other hand, both free gefitinib and GFB/FCPP-NEMs showed a significant dose- dependent cytotoxicity against A549 cells after incubation for $48 \mathrm{~h}$ (Figure $8 \mathrm{~A}$ ). The $50 \%$ inhibitory concentrations $\left(\mathrm{IC}_{50}\right.$ ) for free gefitinib was $8.69 \mu \mathrm{g} / \mathrm{mL}$, while that for GFB/FCPP-NEMs was decreased by 3.82 -fold to $2.27 \mu \mathrm{g} / \mathrm{mL}$, compared to that of free gefitinib. After encapsulation of gefitinib into FCPP-NEMs, the enhanced cytotoxicity of gefitinib can be mainly attributed to their efficient cellular internalization and $\mathrm{pH}$-dependent prolonged release effect resulting from the encapsulation by FCPP-NEMs. As previously reported, encapsulation of chemotherapeutic agents into NPs or MCs is an effective approach to improve their intracellular delivery, and thereby, enhance their antitumor efficacy $[14,16]$. For example, Cosco et al. reported that CS-coated PLGA complexes increased the tumor cells uptake of miR-34a, which led to a significant decrease in tumor cell viability [36]. Similarly, GFB/FCPP-NEMs showed an enhanced cytotoxicity to PC-9 cells at the tested concentrations compared with free gefitinib (Figure $8 \mathrm{~B}$ ). The $\mathrm{IC}_{50}$ for free gefitinib and GFB/FCPP- NEMs was $10.09 \mu \mathrm{g} / \mathrm{mL}$ and $4.52 \mu \mathrm{g} / \mathrm{mL}$, respectively. Thus, the prepared FCPP-NEMs system is a promising multifunctional carrier system for improving the antitumor effects of the chemotherapeutic agent gefitinib. 


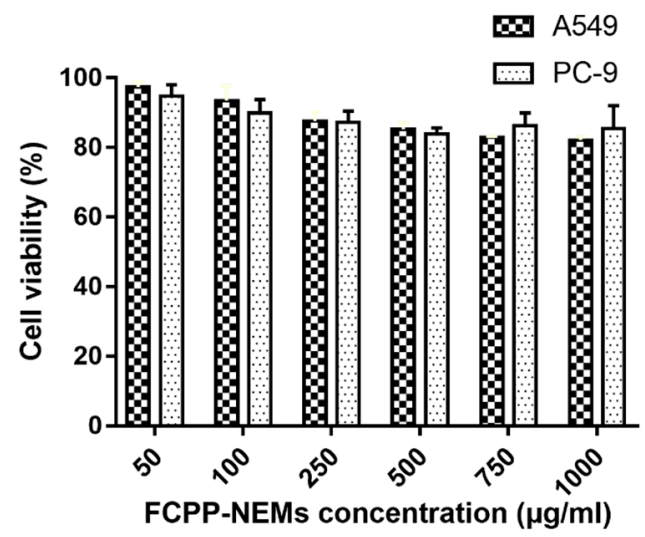

Figure 7. Effect of FCPP-NEMs on A549 and PC-9 cells at various concentrations $(n=6)$.

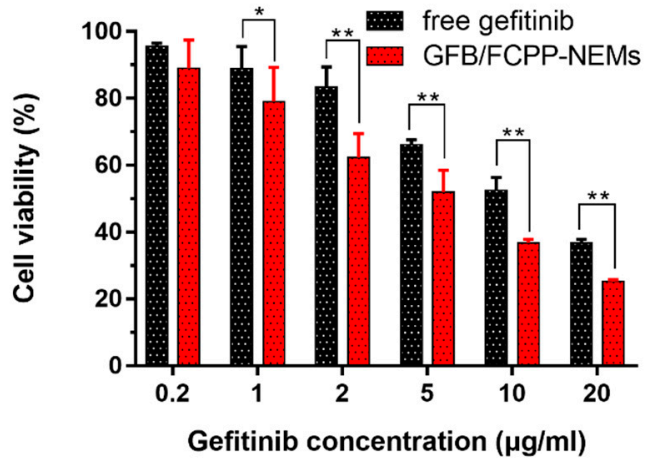

(A)

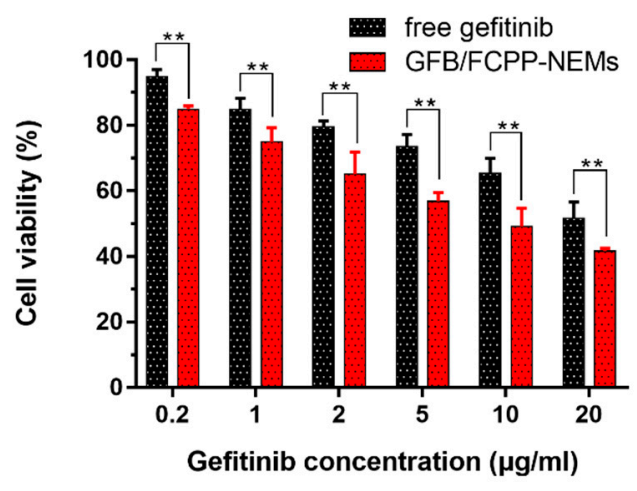

(B)

Figure 8. Effect of free gefitinib and GFB/FCPP-NEMs on A549 cells (A) and PC-9 cells (B) viability at various concentrations $(n=6){ }^{*} p<0.05,{ }^{* *} p<0.01$.

\subsection{Physicochemical Stability}

The particle size, crystallinity, drug content, and related substance of gefitinib were used to evaluate the physicochemical stability of GFB/FCPP-NEMs after storage for 3 months. As shown in Table S3, there were no obvious differences in particle size, drug content, and related substances of gefitinib for the sample of GFB/FCPP-NEMs before and after storage. In addition, the endothermic peak of crystalline gefitinib also cannot be observed in the DSC thermogram of GFB/FCPP-NEMs, suggesting that the prepared GFB/FCPP-NEMs also existed in an amorphous state after storage (Figure S4). Taken together, the GFB/FCPP-NEMs showed an excellent physicochemical stability.

\section{Conclusions}

In this study, a novel FCPP-NEMs-based chemotherapeutic drug delivery system for targeted treatment of lung tumors was designed to afford an FA receptor-mediated tumor targeting property, facilitated intracellular accumulation, $\mathrm{pH}$-triggered sustained release, and enhanced cytotoxicity of the chemotherapeutic agent gefitinib. The prepared GFB/FCPP-NEMs were nearly spherical capsules with a median diameter of around $2.2 \mu \mathrm{m}$ and a highly porous surface. GFB/FCPP-NEMs can quickly dissociate into their primary particles, GFB/FCPP NPs, after hydration. Besides providing $\mathrm{pH}-$ triggered prolonged gefitinib release, the GFB/FCPP-NEMs can facilitate the uptake of the encapsulated cargo by tumor cells. Therefore, the use of GFB/FCPP-NEMs results in a significant improvement in the cytotoxicity of gefitinib compared with the free drug. This study suggests that the developed FCPP-NEMs is a promising carrier system for potential lung tumor-targeted delivery of gefitinib, which is anticipated to improve the antitumor effects of gefitinib and reduce its systemic side effects. 
Supplementary Materials: The following are available online at http://www.mdpi.com/1999-4923/11/9/469/s1, Figure S1: SEM photograph of gefitinib crystals, Figure S2: FT-IR spectra of (a) FA-conjugated CS, (b) FA, and (c) CS, Figure S3: SEM photograph of GFB/FCPP-NEMs after drug release for $2 \mathrm{~h}$, Figure S4: DSC thermogram of GFB/FCPP-NEMs after storage for 3 months, Table S1: Model parameters of the tested samples at pH 5.0, Table S2: Model parameters of the tested samples at pH 7.4, Table S3: Median diameter, span, related substances of gefitinib, and drug content of GFB/FCPP-NEMs before and after storage for 3 months $(n=3)$.

Author Contributions: Conceptualization, X.L. and Y.Z.; methodology, X.L. and Y.Z.; software, X.L. and Z.L.; validation, X.L. and Y.Z.; formal analysis, X.L., J.W., S.L. and Z.Z.; writing-original draft preparation, X.L.; writing-review and editing, X.L. and Y.Z.

Funding: This work was financially supported by the Natural Science Foundation of Jiangsu Province of China (grant number BK20140221).

Acknowledgments: The authors would like to thank the pharmacy students Hongtao Wang, Chan Wang, and Fang Wang who helped in the cell uptake and drug release experiments. The authors would also like to thank the Jiangsu Key Laboratory of New Drug Research and Clinical Pharmacy and the Resource Sharing Platform for Large-scale Instrument and Equipment at Xuzhou Medical University for use of its resources.

Conflicts of Interest: The authors declare no conflict of interest.

\section{References}

1. He, J.; Hu, Y.; Hu, M.; Li, B. Development of PD-1/PD-L1 pathway in tumor immune microenvironment and treatment for non-small-cell lung cancer. Sci. Rep. 2015, 5, 13110. [CrossRef] [PubMed]

2. Brahmer, J.; Reckamp, K.L.; Baas, P.; Crino, L.; Eberhardt, W.E.; Poddubskaya, E.; Antonia, S.; Pluzanski, A.; Vokes, E.E.; Holgado, E.; et al. Nivolumab versus docetaxel in advanced squamous-cell non-small-cell lung cancer. N. Engl. J. Med. 2015, 373, 123-135. [CrossRef] [PubMed]

3. Herbst, R.S.; Giaccone, G.; Schiller, J.H.; Natale, R.B.; Miller, V.; Manegold, C.; Scagliotti, G.; Rosell, R.; Oliff, I.; Revves, A.J.; et al. Gefitinib in combination with paclitaxel and carboplatin in advanced non-small-cell lung cancer: A phase III trial-INTACT 2. J. Clin. Oncol. 2004, 22, 785-794. [CrossRef] [PubMed]

4. Blanco, E.; Shen, H.; Ferrari, M. Principles of nanoparticle design for overcoming biological barriers to drug delivery. Nat. Biotechnol. 2015, 33, 941-951. [CrossRef] [PubMed]

5. Sordella, R.; Bell, D.W.; Haber, D.A.; Settleman, J. Gefitinib-sensitizing EGFR mutations in lung cancer activate anti-apoptotic pathways. Science 2004, 305, 1163-1167. [CrossRef] [PubMed]

6. Stuurman, F.E.; Nuijen, B.; Beijnen, J.H.; Schellens, J.H. Oral anticancer drugs: Mechanisms of low bioavailability and strategies for improvement. Clin. Pharmacokinet. 2013, 52, 399-414. [CrossRef] [PubMed]

7. Inoue, A.; Saijo, Y.; Maemondo, M.; Gomi, K.; Tokue, Y.; Kimura, Y.; Ebina, M.; Kikuchi, T.; Moriya, T.; Nukiwa, T. Severe acute interstitial pneumonia and gefitinib. Lancet 2003, 361, 137-139. [CrossRef]

8. Yang, Y.; Bajaj, N.; Xu, P.; Ohn, K.; Tsifansky, M.D.; Yeo, Y. Development of highly porous large PLGA microparticles for pulmonary drug delivery. Biomaterials 2009, 30, 1947-1953. [CrossRef] [PubMed]

9. Hamdy, S.; Haddadi, A.; Hung, R.W.; Lavasanifar, A. Targeting dendritic cells with nano-particulate PLGA cancer vaccine formulations. Adv. Drug Deliv. Rev. 2011, 63, 943-955. [CrossRef]

10. Bowerman, C.J.; Byrne, J.D.; Chu, K.S.; Schorzman, A.N.; Keeler, A.W.; Sherwood, C.A.; Perry, J.L.; Luft, J.C.; Darr, D.B.; Deal, A.M.; et al. Docetaxel-loaded PLGA nanoparticles improve efficacy in taxane-resistant triple-negative breast cancer. Nano Lett. 2017, 17, 242-248. [CrossRef]

11. Yamamoto, H.; Kuno, Y.; Sugimoto, S.; Takeuchi, H.; Kawashima, Y. Surface-modified PLGA nanosphere with chitosan improved pulmonary delivery of calcitonin by mucoadhesion and opening of the intercellular tight junctions. J. Control. Release 2005, 102, 373-381. [CrossRef] [PubMed]

12. Sheng, J.; Han, L.; Qin, J.; Ru, G.; Li, R.; Wu, L.; Cui, D.; Yang, P.; He, Y.; Wang, J. N-trimethyl chitosan chloride-coated PLGA nanoparticles overcoming multiple barriers to oral insulin absorption. ACS Appl. Mater. Interfaces 2015, 7, 15430-15441. [CrossRef] [PubMed]

13. Dhas, N.L.; Ige, P.P.; Kudarha, R.R. Design, optimization and in-vitro study of folic acid conjugated-chitosan functionalized PLGA nanoparticle for delivery of bicalutamide in prostate cancer. Powder Technol. 2015, 283, 234-245. [CrossRef]

14. Zhu, H.; Chen, H.; Zeng, X.; Wang, Z.; Zhang, X.; Wu, Y.; Gao, Y.; Zhang, J.; Liu, K.; Liu, R.; et al. Co-delivery of chemotherapeutic drugs with vitamin E TPGS by porous PLGA nanoparticles for enhanced chemotherapy against multi-drug resistance. Biomaterials 2014, 35, 2391-2400. [CrossRef] [PubMed] 
15. Cheng, W.; Nie, J.; Xu, L.; Liang, C.; Peng, Y.; Liu, G.; Wang, T.; Mei, L.; Huang, L.; Zeng, X. pH-Sensitive delivery vehicle based on folic acid-conjugated polydopamine- modified mesoporous silica nanoparticles for targeted cancer therapy. ACS Appl. Mater. Interfaces 2017, 9, 18462-18473. [CrossRef] [PubMed]

16. Brannon-Peppas, L.; Blanchette, J.O. Nanoparticle and targeted systems for cancer therapy. Adv. Drug Deliv. Rev. 2012, 64, 206-212. [CrossRef]

17. Batrakova, E.V.; Kabanov, A.V. Pluronic block copolymers: Evolution of drug delivery concept from inert nanocarriers to biological response modifiers. J. Control. Release 2008, 130, 98-106. [CrossRef] [PubMed]

18. Shiehzadeh, F.; Tafaghodi, M. Dry powder form of polymeric nanoparticles for pulmonary drug delivery. Curr. Pharm. Des. 2016, 22, 2549-2560. [CrossRef] [PubMed]

19. Jesenak, M.; Urbancikova, I.; Banovcin, P. Respiratory tract infections and the role of biologically active polysaccharides in their management and prevention. Nutrients 2017, 9, 779. [CrossRef] [PubMed]

20. Roudi, R.; Mohammadi, S.R.; Roudbary, M.; Mohsenzadegan, M. Lung cancer and beta-glucans: Review of potential therapeutic applications. Investig. New Drugs 2017, 35, 509-517. [CrossRef] [PubMed]

21. Francioso, A.; Cossi, R.; Fanelli, S.; Mastromarino, P.; Mosca, L. Studies on trans-resveratrol/carboxymethylated $(1,3 / 1,6)$-beta-d-glucan association for aerosol pharmaceutical applications. Int. J. Mol. Sci. 2017, 18, 967. [CrossRef] [PubMed]

22. Yang, Y.Y.; Chia, H.H.; Chung, T.S. Effect of preparation temperature on the characteristics and release profiles of PLGA microspheres containing protein fabricated by double-emulsion solvent extraction/evaporation method. J. Control. Release 2000, 69, 81-96. [CrossRef]

23. Depan, D.; Shah, J.; Misra, R.D.K. Controlled release of drug from folate-decorated and graphene mediated drug delivery system: Synthesis, loading efficiency, and drug release response. Mater. Sci. Eng. 2011, 31, 1305-1312. [CrossRef]

24. Siepmann, J.; Siepmann, F. Mathematical modeling of drug delivery. Int. J. Pharm. 2008, 364, 328-343. [CrossRef] [PubMed]

25. Pakulska, M.M.; Donaghue, I.E.; Obermeyer, J.M.; Tuladhar, A.; McLaughlin, C.K.; Shendruk, T.N.; Shoichet, M.S. Encapsulation-free controlled release: Electrostaticadsorption eliminates the need for protein encapsulation in PLGA nanoparticles. Sci. Adv. 2016, 2, e1600519. [CrossRef] [PubMed]

26. Zhang, Y.; Dong, K.; Wang, F.; Wang, H.; Wang, J.; Jiang, Z.; Diao, S. Three dimensional macroporous hydroxyapatite/chitosan foam-supported polymer micelles for enhanced oral delivery of poorly soluble drugs. Colloids Surf. B 2018, 170, 497-504. [CrossRef] [PubMed]

27. Liu, G.; Lin, Q.; Huang, Y.; Guan, G.; Jiang, Y. Tailoring the particle microstructures of gefitinib by supercritical $\mathrm{CO}_{2}$ anti-solvent process. J. CO2 Util. 2017, 20, 43-51. [CrossRef]

28. Peer, D.; Karp, J.M.; Hong, S.; Farokhzad, O.C.; Margalit, R.; Langer, R. Nanocarriers as an emerging platform for cancer therapy. Nat. Nanotechnol. 2007, 2, 751-760. [CrossRef] [PubMed]

29. Park, J.H.; Saravanakumar, G.; Kim, K.; Kwon, I.C. Targeted delivery of low molecular drugs using chitosan and its derivatives. Adv. Drug Deliv. Rev. 2010, 62, 28-41. [CrossRef] [PubMed]

30. Zhao, Y.; Luo, Z.; Li, M.; Qu, Q.; Ma, X.; Yu, S.H.; Zhao, Y. A preloaded amorphous calcium carbonate/doxorubicin@silica nanoreactor for $\mathrm{pH}$-responsive delivery of an anticancer drug. Angew. Chem. Int. Ed. Engl. 2015, 54, 919-922. [CrossRef] [PubMed]

31. Liu, J.; Luo, Z.; Zhang, J.; Luo, T.; Zhou, J.; Zhao, X.; Cai, K. Hollow mesoporous silica nanoparticles facilitated drug delivery via cascade $\mathrm{pH}$ stimuli in tumor microenvironment for tumor therapy. Biomaterials 2016, 83, 51-65. [CrossRef] [PubMed]

32. Cannavà, C.; Tommasini, S.; Stancanelli, R.; Cardile, V.; Cilurzo, F.; Giannone, I.; Puglisi, G.; Ventura, C.A. Celecoxib-loaded PLGA/cyclodextrin microspheres: Characterization and evaluation of anti-inflammatory activity on human chondrocyte cultures. Colloids Surf. B 2013, 111, 289-296. [CrossRef] [PubMed]

33. Ford Versypt, A.N.; Pack, D.W.; Braatz, R.D. Mathematical modeling of drug delivery from autocatalytically degradable PLGA microspheres-A review. J. Control. Release 2013, 165, 29-37. [CrossRef] [PubMed]

34. Panda, A.; Meena, J.; Katara, R.; Majumdar, D.K. Formulation and characterization of clozapine and risperidone co-entrapped spray-dried PLGA nanoparticles. Pharm. Dev. Technol. 2014, 21, 43-53. [CrossRef] [PubMed] 
35. Dong, S.; Cho, H.J.; Lee, Y.W.; Roman, M. Synthesis and cellular uptake of folic acid-conjugated cellulose nanocrystals for cancer targeting. Biomacromolecules 2014, 15, 1560-1567. [CrossRef] [PubMed]

36. Cosco, D.; Cilurzo, F.; Maiuolo, J.; Federico, C.; Di Martino, M.T.; Cristiano, M.C.; Tassone, P.; Fresta, M.; Paolino, D. Delivery of miR-34a by chitosan/PLGA nanoplexes for the anticancer treatment of multiple myeloma. Sci. Rep. 2015, 5, 17579. [CrossRef] [PubMed]

(C) 2019 by the authors. Licensee MDPI, Basel, Switzerland. This article is an open access article distributed under the terms and conditions of the Creative Commons Attribution (CC BY) license (http://creativecommons.org/licenses/by/4.0/). 